\title{
Shape stability of $\mathrm{TiSi}_{2}$ islands on $\mathrm{Si} \mathrm{(111)}$
}

\author{
W.-C. Yang, H. Ade, and R. J. Nemanich ${ }^{\text {a) }}$ \\ Department of Physics, North Carolina State University, Raleigh, North Carolina 27695-8202
}

(Received 26 September 2003; accepted 3 November 2003)

\begin{abstract}
The evolution of the shape and size of $\mathrm{TiSi}_{2}$ islands on $\mathrm{Si}$ (111) surfaces is explored with real time ultraviolet photoelectron emission microscopy. During continuous deposition of Ti at elevated temperatures, individual islands in a dilute surface distribution grow larger without island-island interactions. As they increase in size, symmetric islands transform into elongated shaped islands with high length-to-width aspect ratios. An extremely elongated island shows a ratio of 85:1 and is $\sim 17 \mu \mathrm{m}$ long and $\sim 0.2 \mu \mathrm{m}$ wide. The individual elongated islands have different widths regardless of their length. The width of the growing islands is determined at the initial transition stage and remains essentially constant with increasing length. We propose that the various widths of the elongated islands are determined by the degree of strain relaxation, possibly through the nucleation of dislocations at the island interface. In addition, it is found that the elongated islands display a prism-like shape or a truncated prism-like shape. We propose that the shape evolution of the elongated islands is related to both strain relaxation and growth kinetics. (c) 2004 American Institute of Physics. [DOI: 10.1063/1.1636526]
\end{abstract}

\section{INTRODUCTION}

The spontaneous formation of three-dimensional (3D) islands is a common growth mode in heteroepitaxial film growth. Recently, island structures have become of interest for the fabrication of quantum dots or nanowires in lithography-free nanoscale devices. Critical issues for potential applications of self-assembled islands are the growth of uniformly organized islands with well-defined shapes and dimensions. To control island morphology, it is necessary to develop an understanding of island evolution mechanisms. In general, the evolution of initially nucleated islands at late stage growth is governed by coarsening processes such as ripening and coalescence. ${ }^{1}$ In this regime, the size and shape of the growing islands are influenced by the island distribution and the crystal orientation. ${ }^{2}$

In some cases, as epitaxial islands grow larger, symmetric or compact islands are transformed into asymmetric shapes which evolve into longer wire-like structures. The growth of these structures can be employed for the fabrication of nanowires. Since the observation of rectangular hut islands during the initial growth of Ge on silicon, ${ }^{3}$ several groups have observed the formation of elongated island structures on silicon surfaces. ${ }^{4-11}$ Recently, network structures of straight nanowires were produced after the deposition of rare earth metal silicides on $\mathrm{Si}(001) .{ }^{9,10}$ It was proposed that the strain anisotropy is the driving force in creating the rectangular islands, ${ }^{9}$ and that the formation of elongated islands is a general feature for strained epitaxial island growth on lattice mismatched substrates. ${ }^{11}$

The shape transition of epitaxial islands on surfaces is fundamentally dependent on the energetics and the kinetic pathways associated with the island evolution. Tersoff and Tromp have proposed that the shape transition of heteroepi-

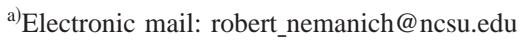

taxial islands into a wire structure is due to the balance between the elastic relaxation and the surface and interface energy. ${ }^{11}$ Below a critical island size, the energy balance favors compact, symmetric islands while above a critical size elongated island shapes are stable. The observation of the shape transition above a critical size was reported by Brongersma et al. during the growth of $\mathrm{CoSi}_{2}$ epitaxial islands on Si (001). ${ }^{8}$ Alternately, a kinetic instability model was proposed that attributed the shape transition to different growth rates of the island facets. ${ }^{12,13}$ This model was postulated to explain the faster growth of end facets in the shape elongation of Ge hut islands on $\mathrm{Si}(001) .{ }^{13}$

However, experimental observations of these shape transitions usually require scanning probe measurements after the growth has proceeded for a fixed time. In order to truly study consecutive shape changes of individual growing islands, it is necessary to employ an in situ, real time microscopy technique, and to image island evolution during continuous deposition at elevated temperatures.

In this study, photoelectron emission microscopy (PEEM) ${ }^{14}$ which has the capability of high-temperature in situ growth and real time imaging, is employed to observe the dynamics of shape transitions of $\mathrm{TiSi}_{2}$ islands on $\mathrm{Si}$ (111) surfaces. The individual islands grow larger without direct interaction with neighboring islands in an initially dilute island distribution. In particular, we observe the elongation of initially nearly circular islands into wire structures and dimensional variation of the evolving islands. Also, we find different cross-sectional shapes of the elongated islands. We propose that the shape evolution of the elongated islands is related to both strain relaxation and growth kinetics.

\section{EXPERIMENT}

The experiments were performed in an UV-PEEM system (Elmitech) with a base pressure $<2 \times 10^{-10}$ Torr. This 
system allows heating of the substrates to $>1200{ }^{\circ} \mathrm{C}$ by filament radiation and electron bombardment from the backside in a sample holder, and the chamber is equipped with a Tifilament deposition source. The UV-light source of the PEEM is a $100 \mathrm{~W}-\mathrm{Hg}$ discharge lamp with an upper cutoff energy near $5.0 \mathrm{eV}$.

Sections of $\mathrm{Si}(111)$ wafers $(n$-type, $P$-doped, resistivity $0.68-0.72 \Omega \mathrm{cm}, 9 \times 9 \mathrm{~mm}^{2}$ ) were employed as substrates. The wafers were cleaned by UV-ozone exposure and then by a HF based spin etch to remove hydrocarbon contaminants and the native oxide layers. After ex situ cleaning, the wafers were mounted to the sample holder and then introduced into the PEEM chamber. Prior to Ti deposition, the wafers were annealed at a temperature of $900{ }^{\circ} \mathrm{C}$ for $10 \mathrm{~min}$. Following the annealing, reflection high-energy electron diffraction displayed a $7 \times 7$ pattern typical of the $\mathrm{Si}$ (111) reconstructed surface.

Titanium (Ti) was deposited in situ from the hot-filament Ti source onto the cleaned surfaces at room temperature. The Ti layer thickness was 1-3 monolayers (ML) with a deposition rate of $1 \mathrm{ML} / \mathrm{min}$. The substrate was subsequently annealed to $1150{ }^{\circ} \mathrm{C}$ with a precision of $\pm 5^{\circ} \mathrm{C}$ and held at the temperature for $30 \mathrm{~min}$. The temperature of the samples was controlled by a thermocouple attached to the sample holder. The actual temperature of the surface was measured with an optical pyrometer. The nucleation and subsequent evolution of Ti silicide islands were observed in real time using the PEEM. The surface density of the islands was controlled by the annealing time. To observe the evolution of the shape and size of individual islands during further growth, Ti evaporation was continued while the surfaces were imaged by PEEM. At a substrate temperature of $1150{ }^{\circ} \mathrm{C}$, Ti was evaporated at a constant growth rate of $\sim 0.3 \mathrm{ML} / \mathrm{min}$.

The images were stored digitally with an image processor and captured on videotape. For analysis of the data, 16 successive images were integrated. The resulting images correspond to an integrated signal of 16/30th of a second. After all processing in the PEEM, the surfaces were examined using an ex situ atomic force microscope (AFM).

\section{RESULTS}

Figure 1 shows a sequence of representative PEEM images of $\mathrm{TiSi}_{2}$ islands on a $\mathrm{Si}$ (111) surface during continuous Ti deposition at $1150{ }^{\circ} \mathrm{C}$. The $\mathrm{TiSi}_{2}$ islands are identified as the bright spots in the images while the exposed underlying $\mathrm{Si}$ surface appears as the darker regions. The PEEM image contrast originates from the photothreshold difference between $\mathrm{TiSi}_{2}(\sim 4.6 \mathrm{eV})$ and $\mathrm{Si}(>5.1 \mathrm{eV})$.

Prior to further Ti deposition, we monitored the surface morphology while about $1 \mathrm{ML}$ of $\mathrm{Ti}$ was deposited on a $\mathrm{Si}$ (111) substrate at room temperature followed by annealing up to $1150^{\circ} \mathrm{C}$. The resultant surface morphology displayed $\mathrm{TiSi}_{2}$ island structures, which is consistent with previous studies. ${ }^{15} 3 \mathrm{D}-\mathrm{TiSi}_{2}$ island formation was observed at $>800{ }^{\circ} \mathrm{C}$. The dimension and surface density of the islands varied with increasing annealing temperature and time. Densely nucleated islands grew larger by island-island interactions through island coarsening processes such as Ostwald
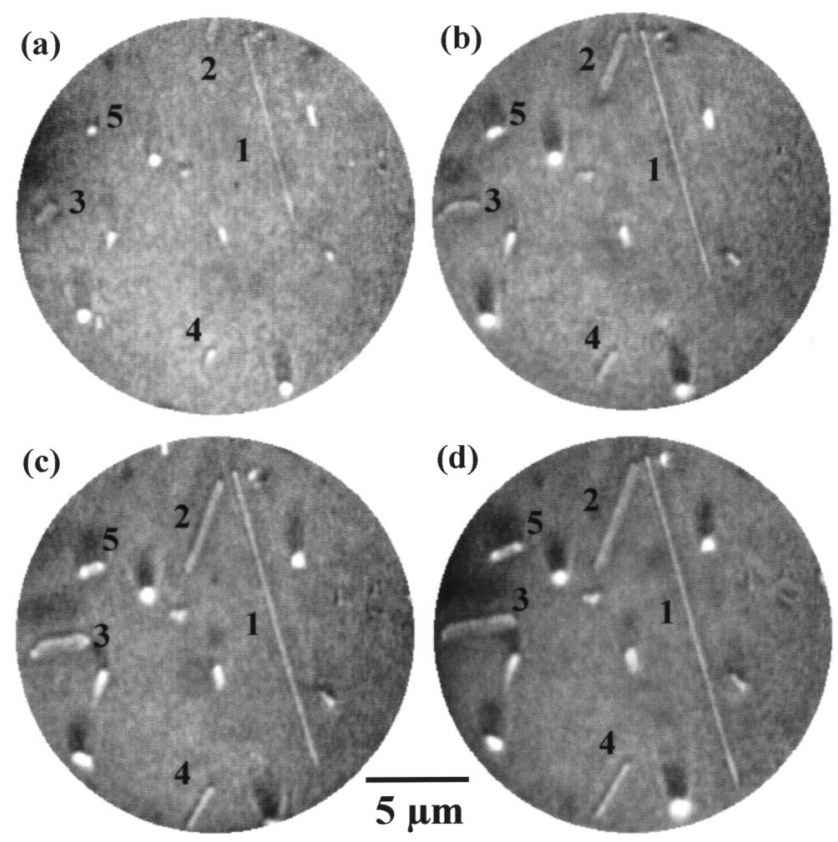

(d)

FIG. 1. Sequential PEEM images of $\mathrm{TiSi}_{2}$ island growth during continuous Ti deposition at $1150{ }^{\circ} \mathrm{C}$. The images were obtained at deposition times of (a) 0 , (b) 5, (c) 10, and (d) $14 \mathrm{~min}$, respectively. The Ti growth rate was 0.3 $\mathrm{ML} / \mathrm{min}$. Identical islands are numbered in (a)-(d).

ripening and coalescence. ${ }^{15}$ Some islands became smaller and disappeared via evaporation without coarsening. With increased annealing time at $1150^{\circ} \mathrm{C}$, the density of islands decreased while the size increased. As a result, annealing for a longer time produced a dilute surface distribution of islands with a low number density (18 islands/227 $\mu \mathrm{m}^{2}$ ) and increased separation [as shown in Fig. 1(a)]. Also, the images indicate that smaller circular islands transform into larger circular, rectangular and wire-like shaped islands.

The progression of images in Fig. 1 reveals several interesting properties of the surface evolution of $\mathrm{TiSi}_{2}$ islands. First, the continuous Ti deposition $\left(0.3 \mathrm{ML} / \mathrm{min}\right.$ at $\left.1150{ }^{\circ} \mathrm{C}\right)$ on the surface with a dilute distribution of circular or compact islands led to larger islands and an unchanged island density [18 islands/227 $\mu \mathrm{m}^{2}$ in Figs. 1 (a) -1 (d)]. Under these conditions, we did not observe additional island nucleation or island disappearance through coarsening processes. Instead, the existing islands grew through absorption of the surface Ti adatoms (supplied from the Ti flux) and reaction with $\mathrm{Si}$ from the surface. This indicates that the arriving $\mathrm{Ti}$ adatoms diffuse rapidly on the surface, and have a low probability of forming additional critical nuclei. In addition, the deposited flux balances any atom loss processes for all of the islands in the image sequence.

Second, the growing islands (as numbered in Fig. 1) show a shape evolution. The islands grow larger in one direction (length) while remaining essentially constant in the perpendicular direction (width). With further growth (or time), the length-to-width aspect ratios of each island increase significantly. An extremely elongated island (island 1) shows an aspect ratio of 85:1 (length: $17 \mu \mathrm{m}$ and width: 0.2 $\mu \mathrm{m})$. This island had already formed an elongated wire shape 


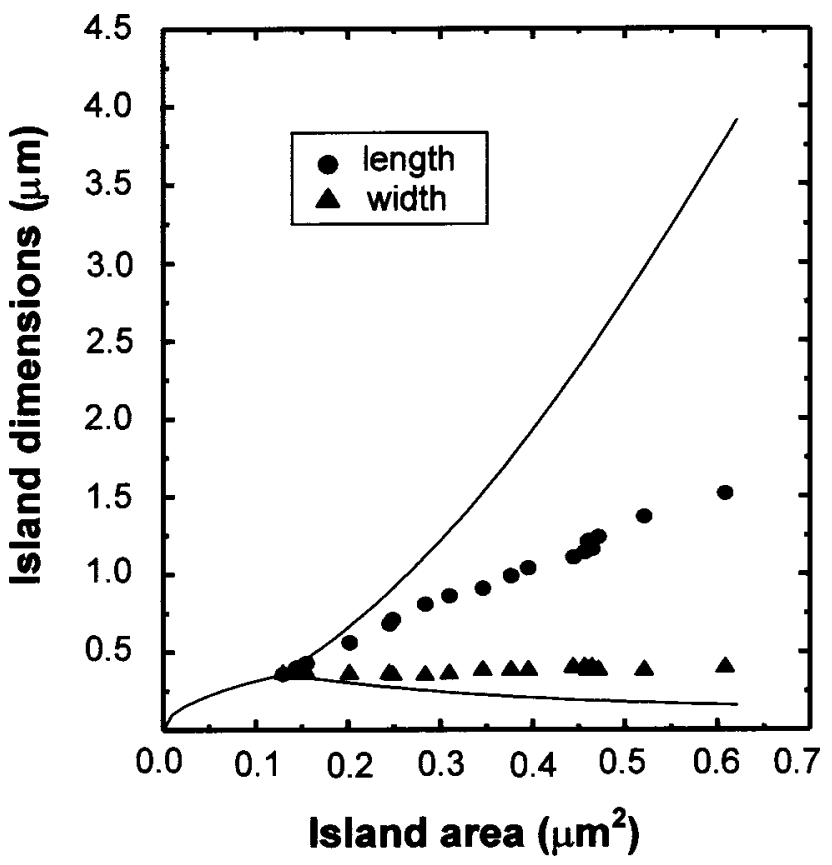

FIG. 2. Length and width of island 5 vs the island surface area. The solid lines represent a theoretical expectation of the dimension variation with a critical size, $e \alpha_{0}=0.36 \mu \mathrm{m}$ (see Ref. 11). This critical size is assumed to be the width of island 5 at the initial growth.

before the continuous deposition. We also observed a specific island that undergoes a shape transition from circular or compact to elongated morphology (island 5). With time, the nearly circular island (island 5) grows through a transition to an elongated shape. Figure 2 shows the evolution of the dimensions of the island during further growth with deposition (time). The initial width of $\sim 0.36 \mu \mathrm{m}$ remained constant while the length increased from 0.36 to $1.5 \mu \mathrm{m}$.

Third, the individual elongated islands have different widths regardless of their length. In PEEM images obtained from other regions of the same surfaces, we found that the islands have various widths [as shown in Fig. 3(a)]; in this group the island widths ranged from $\sim 230 \mathrm{~nm}$ (island 1) to $\sim 1860 \mathrm{~nm}$ (island 5). The width variation was not correlated with the length variation. Also, we found that the elongated
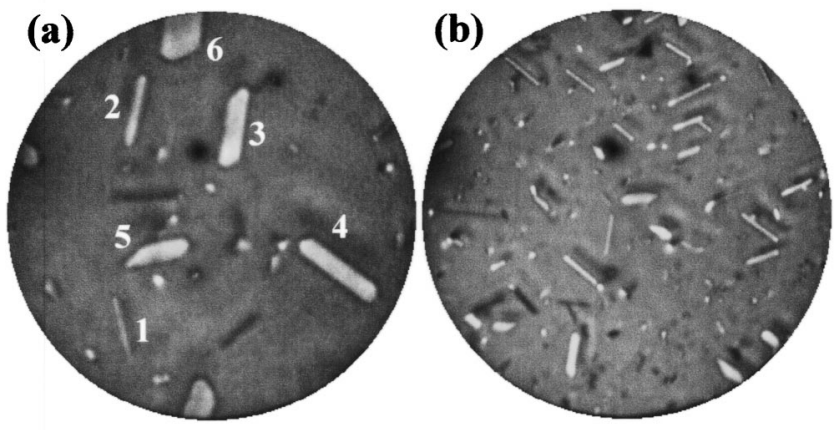

FIG. 3. PEEM images obtained from other regions of the surface at room temperature after finishing island growth. The field of view for the images is 20 and $50 \mu \mathrm{m}$ in (a) and (b), respectively. The measured width of each elongated island in (a): island $1(230 \mathrm{~nm})$, island $2(460 \mathrm{~nm})$, island $3(910$ $\mathrm{nm})$, island $4(900 \mathrm{~nm})$, island $5(920 \mathrm{~nm})$, and island $6(1860 \mathrm{~nm})$. (a)

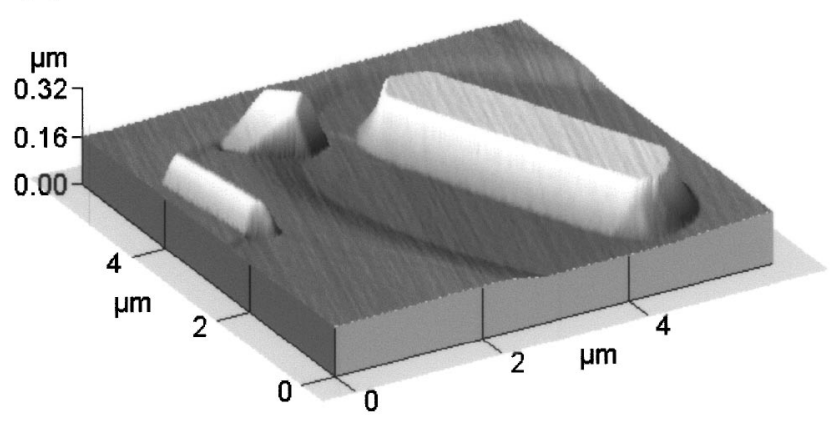

(b)

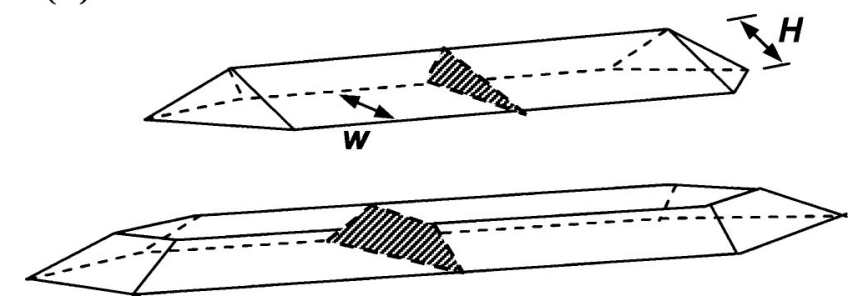

FIG. 4. (a) $3 \mathrm{D}$ AFM images of the $\mathrm{TiSi}_{2}$ islands after cooling to room temperature. (b) Schematic of the two kinds of the elongated islands with hexagon basis.

islands align along similar directions. A larger field of view indicates that the elongated islands grew predominantly along three axes at $\sim 60^{\circ}$ with respect to each other [Fig. 3(b)].

After all processing in the PEEM, the surfaces were examined with ex situ AFM, and the dimensions of the elongated islands were measured. The elongated islands were 1-17 $\mu \mathrm{m}$ long, $0.2-2.5 \mu \mathrm{m}$ wide, and $40-200 \mathrm{~nm}$ high. The measured height of similar width islands did not vary significantly even for a large length. Also, the height was not correlated with the different widths. Rather, the width variation was related to different cross-sectional shapes of the elongated islands. A 3D AFM image displays two neighboring elongated islands of two different shape types [Fig. 4(a)]. The islands were faceted, indicating that they are grown epitaxially. The wider island displays a flat top and trapezoidal cross section, while the narrow island has a peaked top and triangular cross section [Fig. 4(b)].

To compare the apparent height-to-width aspect ratios of the two types of islands, we measured the height and width of 20 islands for each type obtained from AFM images of a single surface. Figure 5 displays a scatter plot of the height/ width ratio versus their widths. We note that trapezoidal islands were wider and had lower $H / W$ values while triangular islands were narrower and had greater $H / W$ values. In addition, the contact angle of the islands was measured with respect to the substrate. The average contact angle of the trapezoidal islands was $21.5^{\circ} \pm 2.8^{\circ}$ which was smaller than that of triangular islands of $32.7^{\circ} \pm 5.2^{\circ}$. These results indicate that the facet planes of the two types of islands appear to be different. 


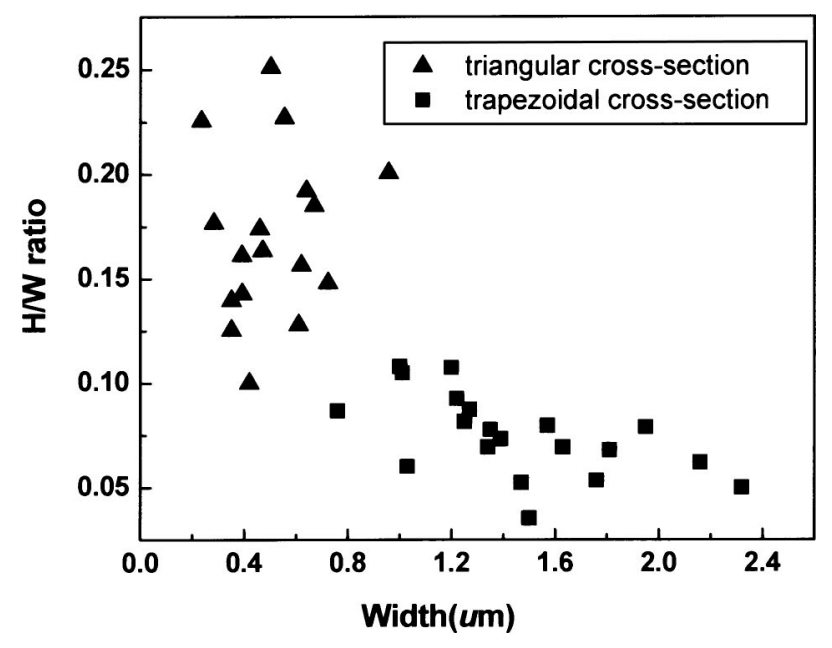

FIG. 5. A scatter plot of height-width ratio vs width of the elongated islands.

\section{DISCUSSION}

Consider mechanisms for the shape transition toward an elongated island structure. Several models have been suggested where the mechanisms are ascribed to strain, energetics, and kinetics. ${ }^{11,13,16}$ Tersoff and Tromp have considered energetics to explain the shape transition and formation of wire structures. ${ }^{11}$ For a strained island of height $h$, width $s$, length $t$, contact angle $\theta$, and volume $V$, they found an expression for the energy per unit volume of the island $(E / V)$. The trade-off between surface energy and strain is obtained through minimization of $E / V$ with respect to $s$ and $t$. This gives $s=t=\alpha_{0}$, which is referred to as the optimal size,

$$
\alpha_{0}=e \phi h \exp (\Gamma / c h),
$$

where $\quad \Gamma=\gamma_{e} \csc \theta-\left(\gamma_{t}+\gamma_{s}+\gamma_{i}\right) \cot \theta, \quad \phi=e^{-3 / 2} \cot \theta, \quad c$ $=\sigma_{b}^{2}(1-\nu) / 2 \pi \mu$. The $\nu, \mu$, and $\sigma_{b}$ are the Poisson ratio, shear modulus of the substrate, and island bulk stress, respectively. The $\gamma_{i}$ is the island-substrate interfacial energy, and $\gamma_{t}, \gamma_{s}$, and $\gamma_{e}$ are the surface energies per unit area of the island's top, the substrate surface, and the island's side facets, respectively.

For an island diameter less than $e \alpha_{0}$, a square or compact island shape is stable. Once the island grows beyond the critical diameter, $e \alpha_{0}$, the square shape becomes unstable and a transition to elongated or rectangular shape takes place. As the island grows further, the length-to-width aspect ratio increases. Our island growth conditions (i.e., well-separated islands and continuous deposition at high temperatures), which were suggested by Tersoff, ${ }^{11}$ are well suited for exploring this model for island elongation. Therefore, the actual dimensional variation of a $\mathrm{TiSi}_{2}$ island can be compared with the analytical prediction. The solid lines of Fig. 2 show the theoretical variation of island 5 in Fig. 1 assuming a critical diameter $\left(e \alpha_{0}\right)$ of $0.36 \mu \mathrm{m}$.

A significant difference between the experimental observation and the analytical expectation is the variation in the width during growth. The analytical calculations anticipate an abrupt transition at a critical size, $e \alpha_{0}$, from square islands to a regime where the islands actively minimize their energy through a shape transition. This results in a quick increase in length and a reduction of the island width, back to the optimum value $\alpha_{0}$. In contrast, our experimental results show that the width of the island remains constant during further growth. Similarly, for the other elongated islands (islands $1-4$ in Fig. 1), the width remained essentially constant with increasing length.

Recently, we observed a similar shape elongation in the growth of $\mathrm{ErSi}_{2}$ islands on $\mathrm{Si}$ (001) surfaces with real time PEEM. ${ }^{17}$ For continuous Er deposition, the length of the elongated islands increased while the width remained constant. Also, a similar development of the shape elongation in the growth of Ge (hut) islands on a $\mathrm{Si}$ surface has been observed with real time scanning tunneling microscopy (STM). ${ }^{12}$ Under high temperature deposition of $\mathrm{Ge}$, the length of the islands became longer while the width did not change. The island elongation was explained by a kinetic instability leading to facet growth, as proposed by Jesson et $a l .{ }^{13}$ In this model, the growth rate of edge facets on the elongated islands was slower than that of end facets due to strain energy considerations. This leads to a further increase in length and a constant width which is determined at an early transition stage.

Under high temperature Ti deposition, due to a low solubility of $\mathrm{Ti}$ in $\mathrm{Si}^{18} \mathrm{Ti}$ adatoms move freely on a $\mathrm{Si}$ surface until the growing islands capture them at its circumferences. The Ti adatoms attach not only to the ends of the elongated islands but also to the edges. The adatoms attached at the edges diffuse to the ends since an energy barrier for the nucleation of a new adatom layer is larger at the edges. ${ }^{12}$ It is noted that the surface concentration of the Si thermal adatoms detached from the step edges at $1150{ }^{\circ} \mathrm{C}$ is $\sim 0.05$ ML. ${ }^{19}$ Thus, the silicide forms from the Si flux, which originates from nearby step edges or through reaction with the substrate, leading to the further elongation of the islands. Therefore, both energetics and growth kinetics contribute to the shape transition and elongation of the $\mathrm{TiSi}_{2}$ islands.

It is interesting to note that the individual elongated islands have different widths, as shown in Fig. 3. The observation of different island widths seems inconsistent with the energetics model where a single width would be preferred. ${ }^{11}$ According to Eq. (1), the critical width would be determined by the material parameters (surface and interface energy, $\Gamma$, and bulk strain energy, $c$ ) and the height of the island. As Tersoff pointed out, strain relaxation in the islands can lead to an increase in the critical size, $\alpha_{0}$, with a decrease in the effective value of the bulk stress component, $\sigma_{b}$ of the island material. This effect could account for the observed widths in the $\mathrm{TiSi}_{2}$ islands.

Recently, a discrete width increase in the growth of Ge islands on $\mathrm{Si}$ has been observed with real time transmission electron microscopy (TEM). ${ }^{20}$ The strain relaxation through the nucleation of a dislocation led to a sudden increase in lateral size. Also, it is noted that the kinetic barrier for dislocation nucleation is inversely proportional to the lattice misfit. ${ }^{21}$ This indicates that the large mismatch of $\mathrm{TiSi}_{2}$ islands at $\mathrm{Si}(111)^{22}$ leads to easy nucleation of dislocations in the islands. Thus, every dislocation nucleation in the islands induces wider islands through strain relaxation which results in various widths for the elongated islands. 
It is evident that the width variation of the elongated islands will also produce different 3D structures as shown in Fig. 4. We propose that the strain relaxation in the growing islands may drive the different cross-sectional shapes of the elongated islands. When strained islands increase in width, there will be a relative increase of the elastic energy. However, each dislocation introduced into an island will lower the misfit and the elastic energy of the island. Thus, the dislocation-induced strain relaxation can lead to an increase in width, which lowers the height-to-width aspect ratio, leading to a flattening of the island's top surface. ${ }^{23}$ In contrast, for a given width, an increase in height will contribute to strain relaxation since the strain energy density decreases from the interface to the peak of the island. ${ }^{13}$ Thus, the height-to-width aspect ratio will be relatively larger, leading to peaked islands, as shown in Fig. 5 .

It was observed that the elongated $\mathrm{TiSi}_{2}$ islands were aligned along one of the sides of an equilateral triangle in the PEEM and AFM images. STM and low-energy electron microscopy measurements of $\mathrm{TiSi}_{2}$ wire-like islands grown on $\mathrm{Si}$ (111) displayed that the islands grow parallel to one of the $\langle 1-10\rangle$ directions. ${ }^{24,25}$ From the threefold symmetry of the $\mathrm{Si}$ (111) substrate we expect the islands to grow along the three equivalent directions. Similarly, elongated silicide islands on $\mathrm{Si}(001)$ aligned along one of two equivalent $\langle 110\rangle$ crystal directions. ${ }^{9,10,17}$ The linear growth of the epitaxial islands is related to the lattice mismatch to the substrate. ${ }^{9}$ To minimize the strain energy of elongated islands, the islands grow preferentially along directions with lowest mismatch. The wires align along a major crystallographic axis which is closely lattice matched to the substrate while the direction of the width may be less matched along the perpendicular axis. ${ }^{11}$

However, Stevens et al. ${ }^{26}$ reported that the anisotropic lattice mismatch mechanism ${ }^{9}$ cannot explain the alignment of the elongated Ti silicide islands. TEM measurements displayed that the nanowires grow into the substrate and have a large lattice mismatch in the long direction. It was proposed that the nanowires grow preferentially along the observed directions to attain a low-energy interface with the substrate.

Alternatively, we speculate that the alignment of the elongated islands is related to the direction of the misfit dislocation formation. At an initial stage of island elongation, a misfit dislocation may nucleate along the $\langle 1-10\rangle$ direction at the $\mathrm{TiSi}_{2}$ island interfaces due to the relatively large lattice mismatch to the perpendicular directions. Then, the islands may grow longer along the dislocation direction. This could explain the direction of linear growth of the epitaxial islands regardless of the complex structure of the interface of the islands with the substrate. However, to reinforce this argument, it is necessary to obtain detailed crystallographic and interfacial structures of the elongated islands through crosssection TEM measurements.

\section{CONCLUSION}

We have utilized real time PEEM to observe the surface evolution of $\mathrm{TiSi}_{2}$ elongated islands on $\mathrm{Si}$ (111) during high- temperature deposition of Ti. By monitoring the evolution of individual islands in a dilute surface distribution, we found a spontaneous shape transition from compact to elongated islands. The widths of the elongated islands were determined at the initial transition stage and remained constant with increasing length. The individual elongated islands have different widths regardless of their length. We suggest that the islands with the narrower width are more highly strained at the interface while the wider islands are more completely relaxed at the interface. The narrow islands showed a peaked triangular cross section while the wider islands showed a flat topped structure. The formation of the two different shapes is also related to strain relaxation. We propose that both interface strain relaxation and growth kinetics can play significant roles in the shape evolution of the elongated islands.

\section{ACKNOWLEDGMENTS}

The authors gratefully acknowledge the Duke Free Electron Laser Laboratory for access to the OK-4 free electron laser. This work was supported by the NSF under Grant No. DMR 0102652 and the DoD MFEL program as administered by the AFOSR through Contract No. F49620-00-1-0370.

${ }^{1}$ M. Zinke-Allmang, L. C. Feldman, and M. H. Grabow, Surf. Sci. Rep. 16, 377 (1992).

${ }^{2}$ I. Daruka, J. Tersoff, and A.-L. Barabasi, Phys. Rev. Lett. 82, 2753 (1999).

${ }^{3}$ Y.-W. Mo, D. E. Savage, B. S. Swartzentruber, and M. G. Lagally, Phys. Rev. Lett. 65, 1020 (1990).

${ }^{4}$ M. Mundschau, E. Bauer, W. Telieps, and W. Swiech, Surf. Sci. 231, 381 (1989).

${ }^{5}$ K. Sekar, G. Kuri, P. V. Satyam, B. Sundaravel, D. P. Mahapatra, and B. N. Dev, Phys. Rev. B 51, 14330 (1995).

${ }^{6}$ D. Loretto, F. Ross, and C. A. Lucas, Appl. Phys. Lett. 68, 2363 (1996).

${ }^{7}$ K. L. Kavanagh, M. C. Reuter, and R. M. Tromp, J. Cryst. Growth 173, 393 (1997).

${ }^{8}$ S. H. Brongersma, M. R. Castell, D. D. Perovic, and M. Zinke-Allmang, Phys. Rev. Lett. 80, 3795 (1998).

${ }^{9}$ Y. Chen, D. A. A. Ohlber, G. M. Riberiro, Y. A. Chang, and R. S. Williams, Appl. Phys. Lett. 76, 4004 (2000).

${ }^{10}$ J. Nogami, B. Z. Liu, M. V. Katkov, and C. Ohbuchi, Phys. Rev. B 653, 233305 (2001).

${ }^{11}$ J. Tersoff and R. M. Tromp, Phys. Rev. Lett. 70, 2782 (1993).

${ }^{12}$ M. Kastner and B. Voigtlander, Phys. Rev. Lett. 82, 2745 (1999).

${ }^{13}$ D. E. Jesson, G. Chen, K. M. Chen, and S. J. Pennycook, Phys. Rev. Lett. 80, 5156 (1998).

${ }^{14}$ H. Ade et al., Surf. Rev. Lett. 5, 1257 (1998).

${ }^{15}$ W.-C. Yang, M. Zeman, H. Ade, and R. J. Nemanich, Phys. Rev. Lett. 90, 136102 (2003).

${ }^{16}$ Y. W. Zhang, Phys. Rev. B 61, 10388 (2000).

${ }^{17}$ L. Fitting, M. C. Zeman, W.-C. Yang, and R. J. Nemanich, J. Appl. Phys. 93, 4180 (2003).

${ }^{18}$ E. R. Weber, Appl. Phys. A: Solids Surf. A30, 1 (1983).

${ }^{19}$ R. M. Tromp, Phys. Rev. Lett. 81, 1050 (1998).

${ }^{20}$ F. K. GeGoues, M. C. Reuter, J. Tersoff, M. Hammar, and R. M. Tromp, Phys. Rev. Lett. 73, 300 (1994).

${ }^{21}$ J. Tersoff and F. K. LeGoues, Phys. Rev. Lett. 72, 3570 (1994).

${ }^{22}$ M. S. Fung, H. C. Cheng, and L. J. Chen, Appl. Phys. Lett. 47, 1312 (1985).

${ }^{23}$ P. Muller and R. Kern, Surf. Sci. 457, 229 (2000).

${ }^{24}$ K. Ezoe, H. Kuriyama, T. Yamamoto, S. Ohara, and S. Matsumoto, Appl. Surf. Sci. 130, 13 (1998).

${ }^{25}$ P. A. Bennett, B. Ashcroft, Z. He, and R. M. Tromp, J. Vac. Sci. Technol. B 20, 2500 (2002).

${ }^{26}$ M. Stevens, Z. He, D. J. Smith, and P. A. Bennett, J. Appl. Phys. 93, 5670 (2003) 